\title{
Comprehensive Evaluation of Teaching Quality Based on Cluster Analysis and Factor Analysis
}

\author{
Zhang Sumei \\ Department of applied mathematics, School of science, Xi'an university of Post and Telecommunications, Xi'an, \\ 710121, China
}

\begin{abstract}
The purpose of this paper was to provide a method for student evaluations of teaching quality. Firstly, the paper introduced cluster analysis and factor analysis theory from the point of view mathematics. Secondly, the paper established a comprehensive evaluation of teaching quality model based on factor analysis and cluster analysis and discussed the process of the model. At last, as an illustration, data from twenty teachers of Xi' an College of Post and Telecommunications were analyzed and modeled by SPSS software. The results from the example show that the proposed approach is efficient. This paper has provided a reference operation model and method for student evaluation of teaching quality.
\end{abstract}

Index Terms: Cluster analysis; Factor analysis; SPSS; Evaluation; Index system

(C) 2011 Published by MECS Publisher. Selection and/or peer review under responsibility of the International Conference on E-Business System and Education Technology

\section{Introduction}

Evaluation of teaching quality has long been a challenge. The importance placed on teaching quality by promotions committees, institutional expectations for faculty development and the increased emphasis on demonstrating outcomes in undergraduate education has only heightened this challenge [1]. In making an evaluation of teaching quality we need to carry out a number of closely related tasks. One of these tasks is to establish evaluation index system by which students can fairly evaluate teaching quality of a teacher. Generally speaking, there are many indexes in evaluating teaching quality. Undoubtedly, a large quantity of indexes will offer rich information for evaluation of teaching quality, while it to some extent increases complexity of evaluation. Since every index reveals some information of teaching quality in varying degrees, and there is often correlation between the indexes, that is, the information provided by those indexes is overlapping to some extent. So it is essential to design several key factors to summarize information in various aspects and there is no correlation between the index and the next.

Cluster analysis [2-3] is a method of unsupervised learning, and a common technique for statistical data

Corresponding author:

E-mail address:zhanggsumei@sina.com 
analysis used in many fields, including machine learning, data mining, pattern recognition, image analysis and bioinformatics. Cluster analysis includes a broad suite of techniques designed to find groups of similar items within a data set. Partitioning methods divide the data set into a number of groups predesigned by the user. Hierarchical cluster methods produce a hierarchy of clusters from small clusters of very similar items to large clusters that include more dissimilar items. Hierarchical methods usually produce a graphical output known as a dendrogram or tree that shows this hierarchical clustering structure. Cluster analysis can be run in the Q-mode in which clusters of samples are sought or in the R-mode, where clusters of variables are desired. Cluster analysis is usually used together with other statistical method such as factor analysis, discriminant analysis etc.

Factor analysis [2-3] is a statistical method used to describe variability among observed variables in terms of fewer unobserved variables called factors. The observed variables are modeled as linear combinations of the factors, plus "error" terms. The information gained about the interdependencies can be used later to reduce the set of variables in a dataset.

The remainder of this paper proceeds as follows: Section 2 established a comprehensive evaluation of teaching quality model; Section 3 describes the major findings drawn from a survey of twenty teachers of Xi' an College of Post and Telecommunications by using SPSS; Section 4 deals with results and discussion.

\section{Comprehensive evaluation model}

\subsection{Model}

Suppose there are $n$ teachers. And we observe $p$ indexes $X_{1}, X_{2}, \cdots, X_{p}$ (observable random variables) of every teacher, and suppose for some unknown constants $a_{i j}$ and $k$ unobserved random variables $F_{j}$, where $i=1, \cdots, p$ and $j=1, \cdots, k$, where $k<p$, thus we have:

$$
\left(\begin{array}{l}
X_{1} \\
X_{2} \\
\vdots \\
X_{p}
\end{array}\right)=\left(\begin{array}{cccc}
a_{11} & a_{12} & \cdots & a_{1 k} \\
a_{21} & a_{22} & \cdots & a_{2 k} \\
\vdots & \vdots & \cdots & \vdots \\
a_{p 1} & a_{p 2} & \cdots & a_{p k}
\end{array}\right)\left(\begin{array}{l}
F_{1} \\
F_{2} \\
\vdots \\
F_{k}
\end{array}\right)+\left(\begin{array}{l}
\varepsilon_{1} \\
\varepsilon_{2} \\
\vdots \\
\varepsilon_{p}
\end{array}\right) .
$$

Omitting the matrix dimensions for clarity, we have:

$$
X=A F+\varepsilon .
$$

Also we will impose the following assumptions on $F$ and $\varepsilon$.

1) $D(F)=I_{k}$;

2) $D(\varepsilon)=\left(\begin{array}{ccc}\sigma_{1}^{2} & & 0 \\ & \ddots & \\ 0 & & \sigma_{p}^{2}\end{array}\right)$, that is, $\varepsilon_{i}$ is uncorrelated and has different variance.

3) $\operatorname{Cov}(F, \varepsilon)=0$, that is, $F$ and $\varepsilon$ are uncorrelated. 
Any solution for the above set of equations following the constraints for $F$ is called as common factors in the index system, and $A$ as the loading matrix.

We construct linear combinations of indexes $X_{1}, X_{2}, \cdots X_{p}$ as follows:

$$
F_{i}=\beta_{i 1} X_{1}+\beta_{i 2} X_{2}+\cdots+\beta_{i p} X_{p} \quad(i=1,2, \cdots, m)
$$

From (3), we can obtain score on every common factor of every subject.

\subsection{Basic process of comprehensive evaluation of teaching quality model}

The model is realized by SPSS. There are usually five basic steps [4-6].

Step 1. Dividing the data set into a number of groups using cluster analysis;

Step 2. Determining whether original variables are appropriate to factor analysis;

Step 3. Constructing factor variables;

Step 4. Make factor variables more clear using rotation together with cluster analysis.

Step 5. Computing factor scores.

All of steps are realized by SPSS 13.0. Now Let us take evaluation of teaching quality of Xi'an College of post and telecommunications as an example to show how to evaluate comprehensive by cluster analysis and factor analysis theory.

\section{Example}

According to undergraduate teaching assessment, a survey was done by department of applied mathematics and physics of Xi'an College of post and telecommunications. Twelve indexes are used in the questionnaire as follows: 1. clear, nature teaching language and standard mandarin; 2. skilled content of teaching and accurate concept; 3. strong logic and systemic; 4. fair and orderly classroom progress; 5. mobilize effectively the enthusiasm and initiative of students; 6 . regulate effectively the progress of teaching according to feedback of students; 7. attend and finish class on time; 8. standard, near writing on blackboard; 9. references by teachers recommend are helpful to students; 10. mark homework regularly and seriously; 11.answer patiently and particularity questions of students; 12. students have great harvest from this course.

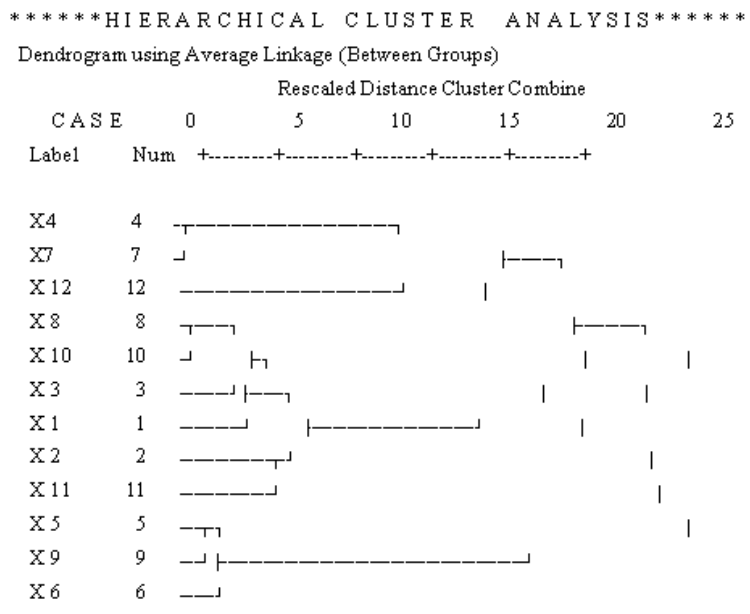

Fig 1. Dendrogram of Cluster Analysis 


\subsection{Data screening}

Here we select randomly twenty teachers whose scores are finished by three hundred students. For convenience of data processing, we name respectively twelve indexes as index 1 , index $2, \ldots$, index 12 , which are twelve observable random variables $X_{1}, X_{2}, \cdots, X_{12}$ in factor analysis model.

\subsection{Running the cluster analysis}

Fig.1 shows dendrogram of cluster analysis. From Fig.1, we can see that all the variables are divided into three groups, of which $\mathrm{X} 4, \mathrm{X} 7$ and $\mathrm{X} 12$ belong to a group; X1, X2, X3, X8, X10 and X11 belong to a group; $\mathrm{X} 5, \mathrm{X} 6$ and $\mathrm{X} 9$ belong to a group.

\subsection{Running the factor analysis}

Table 1 shows several important parts of outputs: the Kaiser-Meyer-Olkin measure of sampling adequacy and Bartlett's test of sphericity. The KMO statistic varies between 0 and 1 . A value close to 1 indicts that patterns of correlation are relatively compact and so factor analysis should yield distinct and reliable factors. Kaiser [7-8] recommends accepting values that greater than 0.5 as acceptable. For these data the value is 0.691 , which falls into the range of being acceptable. Bartlett's measure tests the null hypothesis that original correlation matrix is an identity matrix. For these data, Bartlett's test is highly significant $(\mathrm{p}<0.01)$, and therefore we should be confident that factor analysis is appropriate to these data.

Table $1 \mathrm{KMO}$ and bartlett's test

Kaiser-Meyer-Olkin Measure of Sampling Adequacy $\quad 0.691$

Bartlett's test of sphericity $\quad$ Approx. Chi- Square

df 66

Sig.

.000

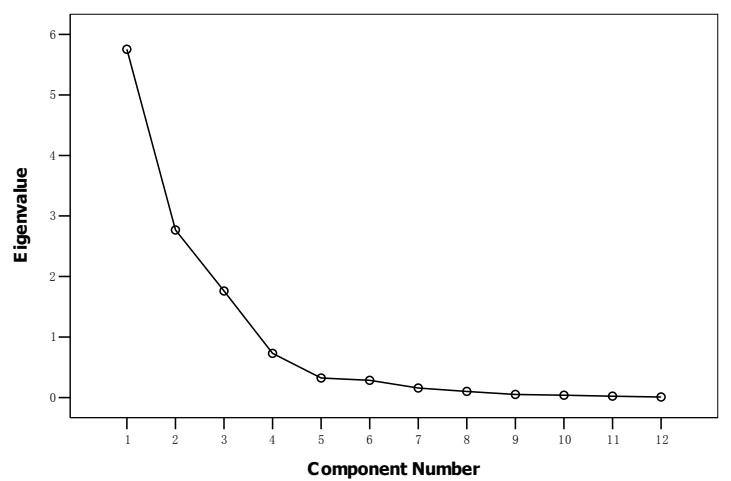

Fig 2. Scree Plot 
The Scree plot (Fig.2) graphs the Eigenvalues against the factor number. We can see these values in the first three columns of the table immediately above. From the fourth factor on, we can see that the line is almost flat, meaning the each successive factor is accounting for smaller and smaller amounts of the total variance. Therefore, we can probably justify retaining either three or four factors which describe effectively original variance.

According to factor analysis and teaching quality evaluation system, the twelve evaluation indexes should be classified into three kinds. We can name respectively them as teaching attitude $\left(F_{1}\right)$, teaching method $\left(F_{2}\right)$ and teaching discipline $\left(F_{3}\right)$, of which $F_{1}$ includes index 1 , index 2 , index 3 , index 8 , index 10 , index $11 ; F_{2}$ includes index 5, index 6, index 9 and $F_{3}$ includes index 4, index 7.

\subsection{Comprehensive evaluation of teaching quality}

Table 2 is the final factor loading matrix, corresponding to matrix $A$ of (3), which indicts the variation of the twelve variables can be explained mainly by the three public factors. According to table II, we can obtain factor analysis model of evaluation of teaching quality as follows:

$$
\left\{\begin{array}{l}
X_{1}=0.781 F_{1}-0.364 F_{2}-0.274 F_{3} \\
X_{2}=0.864 F_{1}-0.014 F_{2}-0.211 F_{3} \\
\cdots \\
X_{12}=0.483 F_{1}+0.216 F_{2}+0.468 F_{3}
\end{array} .\right.
$$

Table 3 is the factor score coefficient matrix and is used to compute factor scores.

Table 2 Component matrix

\begin{tabular}{llll}
\hline & & Component & \\
\cline { 2 - 4 } & \multicolumn{1}{l}{} & $\mathbf{2}$ & -.274 \\
\hline 1 & .781 & -.364 & -.211 \\
3 & .864 & -.014 & -.016 \\
4 & .895 & -.327 & .733 \\
5 & .606 & .044 & -.193 \\
6 & .272 & .913 & -.211 \\
7 & .437 & .827 & .703 \\
8 & .615 & .218 & -.338 \\
9 & .858 & -.273 & -.246 \\
10 & .402 & .853 & -.336 \\
11 & .849 & -.292 & .149 \\
12 & .857 & -.170 & .468
\end{tabular}


Table 3 Factor score coefficient matrix

\begin{tabular}{llll}
\hline & & Component & 3 \\
\cline { 2 - 4 } 1 & \multicolumn{1}{l}{.225} & $\mathbf{2}$ & -.090 \\
2 & .175 & .073 & -.034 \\
3 & .179 & -.062 & .049 \\
4 & -.081 & -.082 & .414 \\
5 & -.043 & .347 & -.026 \\
6 & -.004 & .330 & -.025 \\
7 & -.097 & -.019 & .409 \\
8 & .238 & .009 & -.110 \\
9 & -.004 & .342 & -.044 \\
10 & .239 & .002 & -.111 \\
11 & .115 & -.040 & .135 \\
12 & -.064 & .014 & .282 \\
\hline
\end{tabular}

According to table 3, we can obtain factor score function as follows:

$$
\left\{\begin{array}{l}
F_{1}=0.225 X_{1}+0.175 X_{2}+\cdots-0.064 X_{12} \\
F_{2}=-0.036 X_{1}+0.073 X_{2}+\cdots+0.014 X_{12} \\
F_{3}=-0.090 X_{1}-0.034 X_{2}+\cdots+0.282 X_{12}
\end{array}\right.
$$

According to (3) and (4), SPSS compute automatically factor scores of the three public factors, after inputting evaluating scores of a teacher. We can construct comprehensive evaluation function of teaching quality as follows:

$$
F=0.392 F_{1}+0.242 F_{2}+0.223 F_{3} .
$$

Using this evaluation function, we can obtain comprehensive score of every teacher who is evaluated.

Table 4 Factor score covariance matrix

\begin{tabular}{llll}
\hline & & Component & \\
\cline { 2 - 4 } & 1 & 2 & .000 \\
2 & 1.000 & .000 & .000 \\
3 & .000 & 1.000 & 1.000 \\
\hline
\end{tabular}


Table 4 is the factor score covariance matrix. In the factor analysis model, those public factors obtained by factor analysis should be orthogonal, uncorrelated. From table 4, we can see the three public factors are uncorrelated.

\section{Results and discussion}

This paper has focused on methods used to sustain and encourage tenured faculty to maintain, develop, and improve teaching quality. Through collecting data of twenty teachers of Xi'an College of Post and Telecommunications, we did a case study by SPSS and factor analysis theory. This process has produced a workable model for comprehensive evaluation of teaching quality, through which, we can obtain comprehensive score of every teacher.

Teaching for successful learning cannot occur without high quality evaluation. Evaluation, therefore, needs to be integrated with the process of teaching and learning. Hence, evaluation has to be so designed that it can be used as a powerful means of influencing the quality of what teachers teach and what students learn. But, while doing so special care must be taken to ensure that it is humane and it enables the learner to grow into a responsible and productive citizen. Not only this, evaluation has also to provide constant feedback regarding the effectiveness of course - contents, classroom processes and the growth of individual learners besides the appropriateness of the evaluation procedures. It must, however, be flexible enough to the extent that it can be experimented with and adapted according to the specific situations and needs of the learner groups [9].

Evaluation is an important part of the process of learning, it is about learning from our students, and their learning, and learning about our teaching [10]. These evaluations can enable managers to fine tune aspects of policies, procedures and practices, and thereby enhance the quality of our provision. While quality assurance procedures should continue to draw on a range of processes and expertise, students are clearly key stakeholders who can provide valuable and reliable data to inform quality improvement decisions. Using student evaluations to regularly monitor the teaching quality has proved to be an effective and essential component of the teaching quality management and enhancement process in higher education and the positive impact has been far reaching.

It is worth mentioning that student evaluations of teaching differ from the ideal construct because they are affected by grade leniency and do not reflect learning produced in a course. Even if student evaluations did not suffer from these two deficiencies, student evaluations would assign proper to weight to learning relative to the course experience only under the highly unlikely condition that students place the same weights on these items as the social planner.

\section{References}

[1] Cynthia Silber, Karen Novielli, David Paskin, “ Use of critical incidents to develop a rating form for resident evaluation of faculty teaching”, Medical Education, vol.40, pp. 1201-1208, 2006.

[2] Yu Xiulin,Ren Xuesong, Multivariate Statistical Analysis, China Statistics Press, 1999.

[3] Andy Field, Postgraduate Statistics, Springer, 2005.

[4] W. L. Martinez, A.R. Martinez, Computational Statistics Handbook with MATLAB, Chapman \& Hall/CRC, Boca Raton, 2002.

[5] C. Rose, M.D. Smith, Mathematical Statistics with MATHEMATICA, Springer, 2002.

[6] Jaromír Antoch, "Environment for statistical computing", Computer Science Review, vol.2(2), pp. 113122, Augest 2008.

[7] Yu Jianying, He Xuhong, Statistical analysis and application of SPSS, Beijing: Posts \& Telecommunications Press, 2003.

[8] Kaiser, H. F., “An index of factorial simplicity”, Psychomertrika, vol.39(1), pp.31-35, March 1974. 
[9] Bruce A.Winberg, Belton M.Fleisher and Masanori Hashimoto, "Evaluating teaching in higher education", Journal of Economic Education, vol.40(3), pp.1-40, Summer 2009.

[10] Maureen, Brookes, 'Evaluating the 'student experience': an approach to managing and enhancing quality in higher education", Journal of Hospitality, Leisure, Sport and Tourism Education, vol.2(1), pp.17-26, 2003. 